\title{
Supporting Information: Understanding the Substrate Selectivity and the Product Regioselectivity of Orf2-Catalyzed Aromatic Prenylations
}

\author{
Guanglei Cui and Kenneth M. Merz, Jr.* \\ Quantum Theory Project and Department of Chemistry \\ University of Florida
}

Gainesville, FL 32611-8435

MD Snapshots of the Orf2 Substrate Ternary Complex

The MD snapshots that highlight the transitions between different binding states of 1,6DHN were concatenated into an MPEG animation clip, which can be viewed with Apple Quicktime or Windows Media Player. In the snapshots, the protein fold was rendered as red ribbon with $10 \mathrm{C}$-terminal residues hidden for better viewing. The side chains of residues in the binding site and GPP were rendered as licorice, while the substrate 1,6DHN was drawn as van der Waals spheres, all colored by atom types. During the playback, 1,6-DHN sampled in time $\mathrm{C} 1, \mathrm{C} 2$ and $\mathrm{C} 3$ binding states consecutively. 